\title{
A Novel Mathematical Model of Tumor Growth Kinetics with Allee Effect under Fuzzy Environment
}

Rubeena Khaliq ( $\nabla$ darrubeena63@gmail.com)

BS Abdur Rahman Crescent Engineering College: B S Abdur Rahman Crescent Institute of Science \& Technology

\section{Pervaiz lqbal}

BS Abdur Rahman University: B S Abdur Rahman Crescent Institute of Science \& Technology https://orcid.org/0000-0002-3013-7206

\section{Shahid Ahmad Bhat}

Maharishi Markandeshwar University

\section{Research Article}

Keywords: Tumor growth modeling, Allee effect, Fuzzy differential equation, Population dynamics.

Posted Date: July 27th, 2021

DOI: https://doi.org/10.21203/rs.3.rs-730827/v1

License: (1) (1) This work is licensed under a Creative Commons Attribution 4.0 International License. Read Full License 


\title{
A Novel Mathematical Model of Tumor Growth Kinetics with Allee Effect under Fuzzy Environment
}

\author{
${ }^{1}$ Rubeena Khaliq, ${ }^{2 *}$ Pervaiz Iqbal and ${ }^{1}$ Shahid Ahmad Bhat \\ ${ }^{1,2}$ Deparment of Mathematics \& Actuarial Science, B.S. Abdur Rahman Crescent Institute of Science \& \\ Technology, Chennai, INDIA
}

${ }^{1}$ Department of Mathematics, Maharishi Markandeshwar University, Mullana, Ambala, Haryana, INDIA

\begin{abstract}
Most of the cancer growth models have described the exponential growth patterns at the very initial stage with low cell population density. Eventually, decreasing the tumor growth rate at higher cell population densities because of deficiency in resources such as space and nutrients. However, recent studies at clinical and preclinical investigations of cancer initiation or reappaearance showed a population dynamics evincing that the growth rate increases as cell number increases. Hence, showing behaviour analogous to cooperative mechanism in the ecosystem and ecological effect called Allee effect. Based on these observations with two arguments i.e. change in initial population and growth rate. In this paper, the novel mathematical model of tumor growth kinetics with Allee effect under fuzzy environment is proposed. In this model the Generalized Hukuhara derivative approach is utilized to solve the fuzzy differential equations. Moreover, it is showen that the change in initial value and growth rate affects the cell density with the Allee effect under the fuzzy environment. Finally the superiority of model has been showen with the help of numerical simulation.
\end{abstract}

Keywords: Tumor growth modeling, Allee effect, Fuzzy differential equation, Population dynamics.

\section{Introduction}

Relating cancer to the ecological and evolutionary process, in a stable ecosystem (healthy body), if one of the species (cells) getaway from ecological restraints (immune system) and proliferates speedily, hence destroying the stability, precipitating the extinction of neighbouring

*Corresponding Author

pervaizmaths@gmail.com (Pervaiz Iqbal) 
species and therefore, collapse the ecosystem (disturbs body's balance, cause the death of healthy cells and made the body like acidic) called cancer [1, 2]. The tumor growth cell mechanism depends upon how potentially cells are capable of availing the facilities and how profitably they construct a mechanism to escape the identification and extinction by the defense system of the body [3]. Kirill et. al. [4] highlighted the four prime characteristic features explaining why the cancer is burdensome to cure as 1) Limited information of cellular and tissue level process. 2) Facing challenge to identify and hit cancer cells as they are clone to normal cells. 3) Due to the rapid evolution of cancer cells, it is very hard to overcome the tumor suppressor mechanism. 4) The clonal diversity in heterogeneous makeup thus, permiting for cancer reappearance after remission frequently.

The application of evolutionary modeling to cancer growth has yielded significant results. Despite widespread recognition of cancer as an evolutionary mechanism, little research has been done to characterize cancer's ecological dimensions. Our understanding of ecological processes with respect to tumors, in particular, is minimal [4 - 24]. The logistic model and Gompertz model have been proved the best fit model in describing the tumor dynamics [25]. An ecological effect called as an Allee effect or inverse density dependence is one of the frequent departure from logistic growth and Gompertz growth models [26, 27].

\section{Alley Effect in Tumor Growth}

Warder Clyde discovered the Allee effect first in 1930. It states that the allele effect is a biological occurrence to divulge a high correlation between population size or density and the individual mean population fitness. The population with a peak per capita growth rate at low cell population density corresponds to a phenomenon called Allee effect. It happens when the growth rate per capita rises as the density increases and decreases when a certain level is reached, which is often referred to as the threshold. Variations in various parameters, such as social isolation in small-scale, inbreeding depression, finding a spouse, predator avoidance of defence and food exploitation may all contribute to this effect and the problem of searching indo-individual pairs of low population density species is the main cause of this effect [28 - 35].

Ecologists and mathematicians have since studied this effect in many different situations in different fields. It is assumed, in most models that the growth of cancers by cell-autonomous 
proliferation, evinced as an increase in cell number exponentially at initial and finally restricted by the carrying capacity [36]. However, the Allele effect shows active involvement in tumor growth kinetics by describing the cancer growth at low cell population densities deviates from the exponential growth path $[37,4,38]$. The impact on tumor growth due to the Allee effect through the features of complex models integrating the Allee effect and the implications of the presence of such an effect on the option of the most suitable therapy is described [35].

Allee effect can be induced by several factors mentioned above but among them, potentially relevant to cancer can be a cooperative interaction mechanism [4]. A population that may increase at a medium population density but decreases if the species count is either too small or too large describes strong Allee effect while as a weak Allee Effect represents a population that rises at a low population level with a decreased per capita growth rate, although the growth rate is still positive [39]. A very recent study identified the possible presence of a weak Allee effect in tumor cells. Through a series of in vitro experiments the lower density rate of tumor cell growth was tested with two glioblastoma cell lines, where it was observed that the growth rate increased with population density when the cell density was low while decreasing at higher density. weak Allee effect of cancer cells in cultures probably results from autocrine growth factors, diffuse signaling molecules formed and secreted by cells that promote the growth and proliferation of other cells [40,38]. The mathematical model of a tumor with the week alley effect demonstrates how the intensity of the Allee effect affects the tumor size [41].

\section{Fuzzy Theory and Applications}

The fuzzy mathematical modeling can be used to simulate a variety of real-world phenomena where there are certain complexities due to inexactness and vagueness [42 - 45]. Firstly, the fuzzy concept in 1956, has introduced by Professor Lotfi Ahmad Zadeh, at the University of Berkeley and the ambiguous and impreciseness are dealt with in fuzzy logic. In other words, rather than the standard true/false or 1/0 like Boolean logic, it provides an understanding of real-life problems dependent on degrees of fact. The concept of fuzzy is logic that is used to designate fuzziness rather than logic that is fuzzy. In oncology, related to tumor growth, fuzzy mathematical models have been used by A.M Nasarbadi in 2009 and 2010 in which fuzzy differential equations have been solved for tumor growth solutions [46, 47]. Mahdieh in 2018 described a full fuzzy mathematical model of tumor growth with the help of 
IPD equations [48]. Souza et. al. [49] surfaced out the dynamics of tumor growth using fuzzy theory and fuzzy neotic threshold. Further investigation into fuzzy tumor modeling can be seen in $[50-54]$.

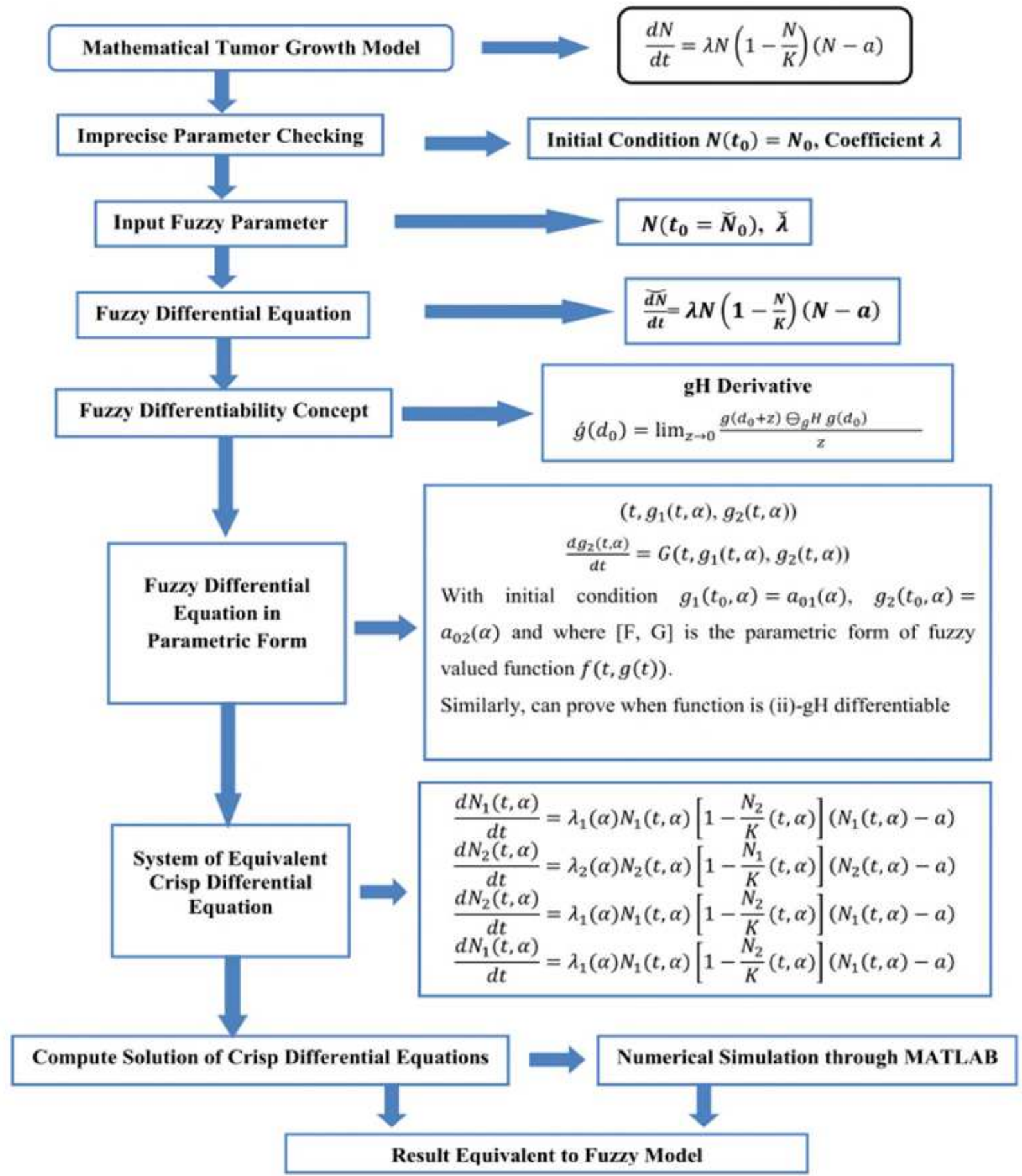

Fig.1. Algorithm and Architecture for Solving Objective of the Study 


\section{Algorithm and Architecture of the work}

This work is aimed to check the behavior of tumor growth with the involvement of the Allee effect in a fuzzy environment with differentiability concepts (Generalized Hukuhara Derivative) in the population growth models. In this work, a fuzzy mathematical model is developed in which fuzziness is implemented in different parameters separately and together to check the tumor growth behaviour. Three cases are discussed as initial condition as fuzzy, coefficient as fuzzy and both as fuzzy in logistic equation with Allee effect in the tumour microenvironment. Numerical simulation is given to support this work. The algorithm and architecture of the proposed work is showen in Fig.1.

\section{Preliminaries}

\subsection{Fuzzy set}

A fuzzy set $\tilde{A}$ in $\Re$ is a set of ordered such that

$$
\tilde{A}=\{(x, \mu \tilde{A}(x)) / x \in \Re\}
$$

Where $\mu \tilde{A}: \Re \rightarrow[0,1]$ and $\mu \tilde{A}(x)$ is called the membership function for the fuzzy set and $\mathfrak{R}$ represents the set of real numbers.

\subsection{Triangular fuzzy number}

A fuzzy number that is represented with three points as follows

$$
\begin{gathered}
\tilde{A}=\left(a_{1}, a_{2}, a_{3}\right),\left(a_{1}<a_{2}<a_{3}\right) \text { and whose membership function is given by } \\
\mu \tilde{A}(x)= \begin{cases}\frac{x-a_{1}}{a_{2}-a_{1}}, a_{1} \leq x \leq a_{2} \\
\frac{a_{3}-x}{a_{3}-a_{2}}, a_{2} \leq x \leq a_{3} \\
0, & \text { otherwise }\end{cases}
\end{gathered}
$$

is called a triangular fuzzy number[55]. 


\section{3. $\alpha$-cut}

$\alpha$-cut of triangular fuzzy number $\tilde{A}=\left(a_{1}, a_{2}, a_{3}\right)$ is given by

$$
A_{\alpha}=\left[a_{1}+\alpha\left(a_{2}-a\right), a_{3}-\alpha\left(a_{3}-a_{2}\right)\right], \forall \alpha \in[0,1]
$$

\subsection{Generalized Hukuhara derivative for fuzzy valued function}

The Generalized Hukuhara derivative for fuzzy valued function $g:(a, b) \rightarrow \Re_{\mathcal{F}}$ at the point $d_{0}$ is defined as

$$
\dot{g}\left(d_{0}\right)=\lim _{z \rightarrow 0} \frac{g\left(d_{0}+z\right) \ominus_{g} H g\left(d_{0}\right)}{z}
$$

If $\dot{g}\left(d_{0} \in \Re_{\mathcal{F}}\right.$ which can be found from (4), we can say that $g(t)$ is generalized Hukuhara derivative at $t_{0}$

The main theory is that if $g(t)$ is (i)-gH differentiable at $d_{0}$ then

$$
\left[\dot{g}\left(d_{0}\right)\right]_{\alpha}=\left[\dot{g}_{1}\left(d_{0}, \alpha\right), \dot{g}_{2}\left(d_{0}, \alpha\right)\right]
$$

And $g(t)$ is (ii)-gH differentiable at $d_{0}$ then

$$
\left[\dot{g}\left(d_{0}\right)\right]_{\alpha}=\left[\dot{g}_{2}\left(d_{0}, \alpha\right), \dot{g}_{1}\left(d_{0}, \alpha\right)\right]
$$

Where the $\alpha$-cut of $g(t)$ is defined as $\left[g_{1}(t, \alpha), g_{2}(t, \alpha)\right]$

Proof: see the paper [56].

\subsection{Characterization Theorem for Differential Equation in Fuzzy Environment}

Consider the fuzzy initial value problem [57] as

$$
\frac{d g(t)}{d t}=f(t, x(t)), \quad t \in\left[t_{0}, T\right]
$$

With initial condition $g\left(t_{0}\right)=a_{0}$ 
Where $f: I \times E \rightarrow E$ is a continuous fuzzy mapping and $a_{0}$ or the coefficient of the differential equation or both are fuzzy numbers. The interval may be like $[0, T]$ for some $T>0$ or $I=$ $[0, \infty)$

Theorem: if $f: I \times E \rightarrow E$ is a continuous fuzzy function such that there exists $k>$ 0suchthat $(f(t, y), f(t, z)) \leq k D(y, z) \forall t \in I, y, z \in E$. Then equation $1,2,3$ has two different solutions namely (i)-gH differentiable solution and (ii)-gH differentiable solution on $I$.

Proof: See paper [58]

The parametric form of derivative of a fuzzy valued function is two types when the function is (i)-gH differentiable and (ii)-gH differentiable [59].

\section{Crisp model Theory}

Population evolution (cancer cells) are best described by Logistic population evolutionary models with the Allee effect as:

$$
\frac{d N}{d t}=\lambda N\left(1-\frac{N}{K}\right)(N-a)
$$

$$
\text { With initial condition } N\left(t_{0}\right)=N_{0}
$$

Where $N$ is the number of cells, $\lambda$ is the total growth rate, $K$ is carrying capacity, $t$ is a time of occurrence and $a$ is an Allee threshold. It is assumed that at time $=0, N=10^{6}$ i.e. one billion cells. It is also assumed that $N<K, \lambda\left(1-\frac{N}{K}\right)$ is per capita logistic growth rate and $\lambda\left(1-\frac{N}{K}\right)(N-a)$ is modified per capita growth rate. However, in the presence of the Allee effect, the per capita growth decreases below a given population size and can be negative below the Allee threshold $(a)$ which eventually may extinct.

\section{Proposed model of Tumor Growth in Fuzzy Environment}

If we consider now equation (5) in the fuzzy environment then three cases arise in (5) as:

\section{a) Fuzziness in the initial condition}


The transformed model using differentiability concept, when $N(t)$ is (i)-gH Differentiable in equation (5)

$$
\begin{aligned}
& \frac{d N_{1}(t, \alpha)}{d t}=\lambda_{1} N_{1}(t, \alpha)\left[1-\frac{N_{2}}{K}(t, \alpha)\right]\left(N_{1}(t, \alpha)-a\right) \\
& \frac{d N_{2}(t, \alpha)}{d t}=\lambda_{2} N_{2}(t, \alpha)\left[1-\frac{N_{1}}{K}(t, \alpha)\right]\left(N_{2}(t, \alpha)-a\right)
\end{aligned}
$$

With initial conditions $N_{1}\left(t_{o}, \alpha\right)=N_{o 1}(\alpha)$ and $N_{2}\left(t_{o}, \alpha\right)=N_{o 2}(\alpha)$

The transformed model using differentiability concept, when $N(t)$ is (ii)-gH Differentiable in equation (5)

$$
\begin{aligned}
& \frac{d N_{2}(t, \alpha)}{d t}=\lambda_{1} N_{1}(t, \alpha)\left[1-\frac{N_{2}}{K}(t, \alpha)\right]\left(N_{1}(t, \alpha)-a\right) \\
& \frac{d N_{2}(t, \alpha)}{d t}=\lambda_{2} N_{2}(t, \alpha)\left[1-\frac{N_{1}}{K}(t, \alpha)\right]\left(N_{2}(t, \alpha)-a\right)
\end{aligned}
$$

With initial conditions $N_{1}\left(t_{o}, \alpha\right)=N_{o 1}(\alpha)$ and $N_{2}\left(t_{o}, \alpha\right)=N_{o 2}(\alpha)$

\section{b) Fuzziness in coefficient}

The transformed model using differentiability concept, when $N(t)$ is (i)-gH Differentiable in equation (5)

$$
\begin{aligned}
& \frac{d N_{1}(t, \alpha)}{d t}=\lambda_{1}(\alpha) N_{1}(t, \alpha)\left[1-\frac{N_{2}}{K}(t, \alpha)\right]\left(N_{1}(t, \alpha)-a\right) \\
& \frac{d N_{2}(t, \alpha)}{d t}=\lambda_{2}(\alpha) N_{1}(t, \alpha)\left[1-\frac{N_{2}}{K}(t, \alpha)\right]\left(N_{1}(t, \alpha)-a\right)
\end{aligned}
$$

With initial conditions $N_{1}\left(t_{o}, \alpha\right)=N_{o}$ and $N_{2}\left(t_{o}, \alpha\right)=N_{o}$

The transformed model using differentiability concept, when $N(t)$ is (ii)-gH Differentiable in equation (5) 


$$
\begin{aligned}
& \frac{d N_{2}(t, \alpha)}{d t}=\lambda_{1}(\alpha) N_{1}(t, \alpha)\left[1-\frac{N_{2}}{K}(t, \alpha)\right]\left(N_{1}(t, \alpha)-a\right) \\
& \frac{d N_{1}(t, \alpha)}{d t}=\lambda_{2}(\alpha) N_{2}(t, \alpha)\left[1-\frac{N_{1}}{K}(t, \alpha)\right]\left(N_{2}(t, \alpha)-a\right)
\end{aligned}
$$

With initial conditions $N_{1}\left(t_{o}, \alpha\right)=N_{o}$ and $N_{2}\left(t_{o}, \alpha\right)=N_{o}$

\section{c) Fuzziness in both initial condition and coefficient}

The transformed model using differentiability concept, when $N(t)$ is (i)-gH Differentiable in equation (5)

$$
\begin{aligned}
& \frac{d N_{1}(t, \alpha)}{d t}=\lambda_{1}(\alpha) N_{1}(t, \alpha)\left[1-\frac{N_{2}}{K}(t, \alpha)\right]\left(N_{1}(t, \alpha)-a\right) \\
& \frac{d N_{2}(t, \alpha)}{d t}=\lambda_{2}(\alpha) N_{2}(t, \alpha)\left[1-\frac{N_{1}}{K}(t, \alpha)\right]\left(N_{2}(t, \alpha)-a\right)
\end{aligned}
$$

With initial conditions $N_{1}\left(t_{o}, \alpha\right)=N_{o 1}(\alpha)$ and $N_{2}\left(t_{o}, \alpha\right)=N_{o 2}(\alpha)$

The transformed model using differentiability concept, when $N(t)$ is (ii)-gH Differentiable in equation (5)

$$
\begin{aligned}
& \frac{d N_{2}(t, \alpha)}{d t}=\lambda_{1}(\alpha) N_{1}(t, \alpha)\left[1-\frac{N_{2}}{K}(t, \alpha)\right]\left(N_{1}(t, \alpha)-a\right) \\
& \frac{d N_{1}(t, \alpha)}{d t}=\lambda_{1}(\alpha) N_{1}(t, \alpha)\left[1-\frac{N_{2}}{K}(t, \alpha)\right]\left(N_{1}(t, \alpha)-a\right)
\end{aligned}
$$

With initial conditions $N_{1}\left(t_{o}, \alpha\right)=N_{o 1}(\alpha)$ and $N_{2}\left(t_{o}, \alpha\right)=N_{o 2}(\alpha)$

\section{Numerical simulation}

\section{Tumor Growth Model including Allee Effect Along with Initial Condition and Coefficients} are both Fuzzy

When $N(t)$ is (i)-gH Differentiable, the model transformed using this differentiability concept as 


$$
\begin{aligned}
& \frac{d N_{1}(t, \alpha)}{d t}=\lambda_{1}(\alpha) N_{1}(t, \alpha)\left[1-\frac{N_{2}}{K}(t, \alpha)\right]\left(N_{1}(t, \alpha)-a\right) \\
& \frac{d N_{2}(t, \alpha)}{d t}=\lambda_{2}(\alpha) N_{2}(t, \alpha)\left[1-\frac{N_{1}}{K}(t, \alpha)\right]\left(N_{2}(t, \alpha)-a\right) \\
& \text { With initial conditions } N_{1}\left(t_{o}, \alpha\right)=N_{o 1}(\alpha) \text { and } N_{2}\left(t_{o}, \alpha\right)=N_{o 2}(\alpha) \\
& \text { Take } N(O)=(0.46,0.51,0.66) \\
& \lambda=(0.016,0.026,0.031) \\
& a=2, K=7, t \in[0,1000] \\
& \frac{d N_{1}(t, \alpha)}{d t}=(0.016+0.01 \alpha) N_{1}(t, \alpha)\left[1-\frac{N_{2}}{7}(t, \alpha)\right]\left(N_{1}(t, \alpha)-2\right) \\
& \frac{d N_{2}(t, \alpha)}{d t}=(0.031-0.005 \alpha) N_{2}(t, \alpha)\left[1-\frac{N_{1}}{7}(t, \alpha)\right]\left(N_{2}(t, \alpha)-2\right)
\end{aligned}
$$

With initial conditions $N_{1}(0, \alpha)=0.46+0.05 \alpha$ and $N_{2}(0, \alpha)=0.66-0.15 \alpha$ 

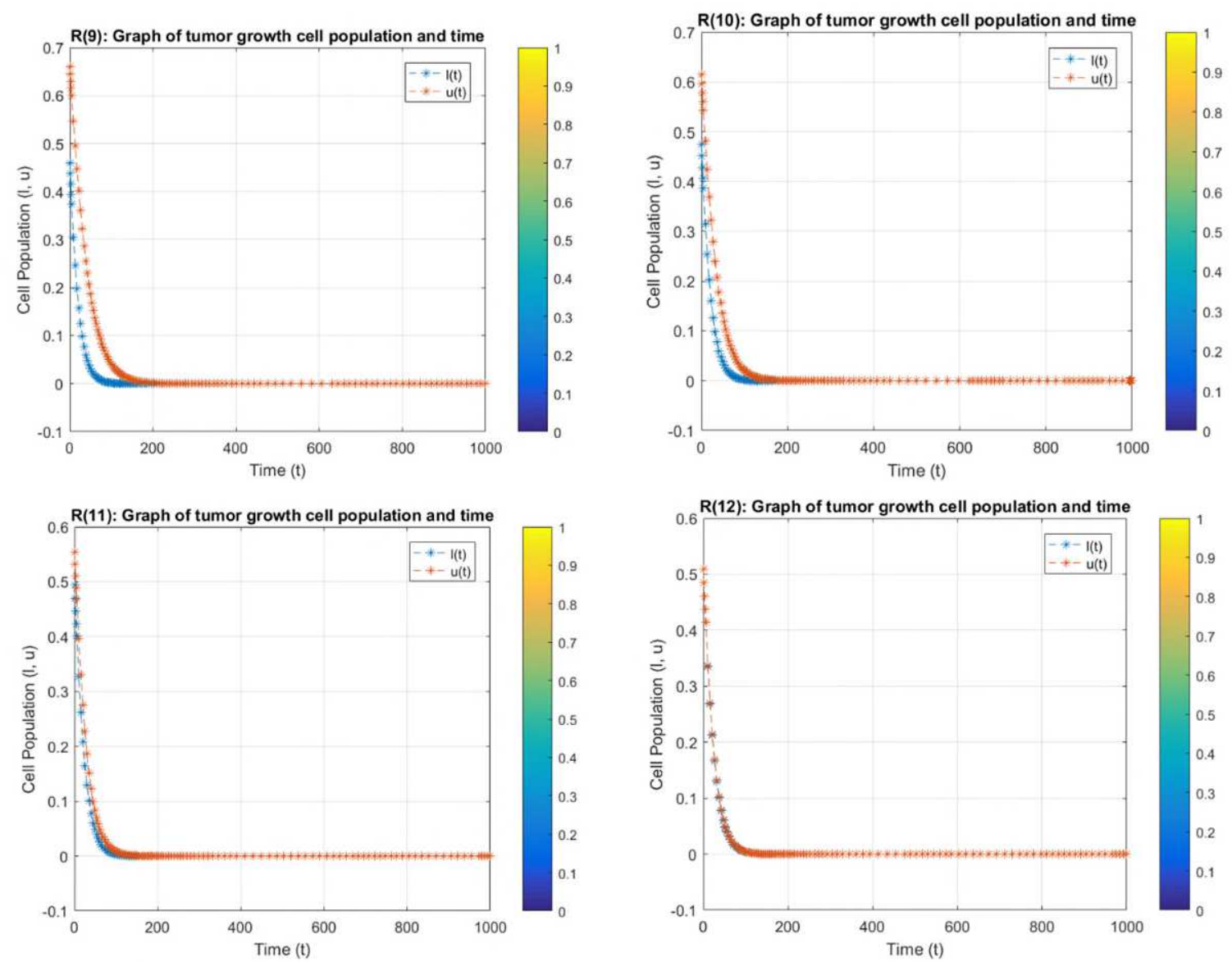

Fig. 2. Numerical simulation for both fuzzy initial condition and coefficient when $N(t)$ is (i)-gH for tumor growth model with Allee effect. For $\alpha=0, \alpha=0.3, \alpha=0.7$ the solution $N_{1}(t, \alpha) \leq$ $N_{2}(t, \alpha)$ for $t \epsilon[0,180]$ and for $\alpha=1$, both $N_{1}(t, \alpha)=N_{2}(t, \alpha)$ for all $t$ and finally approaches to the unstable solution. This figure depicted a Simultaneous graph of the tumor growth model when the Initial value is greater or smaller than the Allee threshold $(a=2)$ and $K=7$.

When $N(t)$ is (ii)-gH Differentiable, the model transformed using this differentiability concept as

$$
\begin{aligned}
& \frac{d N_{2}(t, \alpha)}{d t}=\lambda_{1}(\alpha) N_{1}(t, \alpha)\left[1-\frac{N_{2}}{K}(t, \alpha)\right]\left(N_{1}(t, \alpha)-a\right) \\
& \frac{d N_{1}(t, \alpha)}{d t}=\lambda_{2}(\alpha) N_{2}(t, \alpha)\left[1-\frac{N_{1}}{K}(t, \alpha)\right]\left(N_{2}(t, \alpha)-a\right)
\end{aligned}
$$

With initial conditions $N_{1}\left(t_{o}, \alpha\right)=N_{o 1}(\alpha)$ and $N_{2}\left(t_{o}, \alpha\right)=N_{o 2}(\alpha)$ 


$$
\text { Take } N(O)=(0.46,0.51,0.66)
$$

$$
\lambda=(0.016,0.026,0.031)
$$

$$
a=2, K=7, t \in[0,1000]
$$

$$
\begin{gathered}
\frac{d N_{2}(t, \alpha)}{d t}=(0.016+0.01 \alpha) N_{1}(t, \alpha)\left[1-\frac{N_{2}}{7}(t, \alpha)\right]\left(N_{1}(t, \alpha)-2\right) \\
\frac{d N_{1}(t, \alpha)}{d t}=(0.031-0.005 \alpha) N_{2}(t, \alpha)\left[1-\frac{N_{1}}{7}(t, \alpha)\right]\left(N_{2}(t, \alpha)-2\right)
\end{gathered}
$$

With initial conditions $N_{1}(0, \alpha)=0.46+0.05 \alpha$ and $N_{2}(0, \alpha)=0.66-0.15 \alpha$
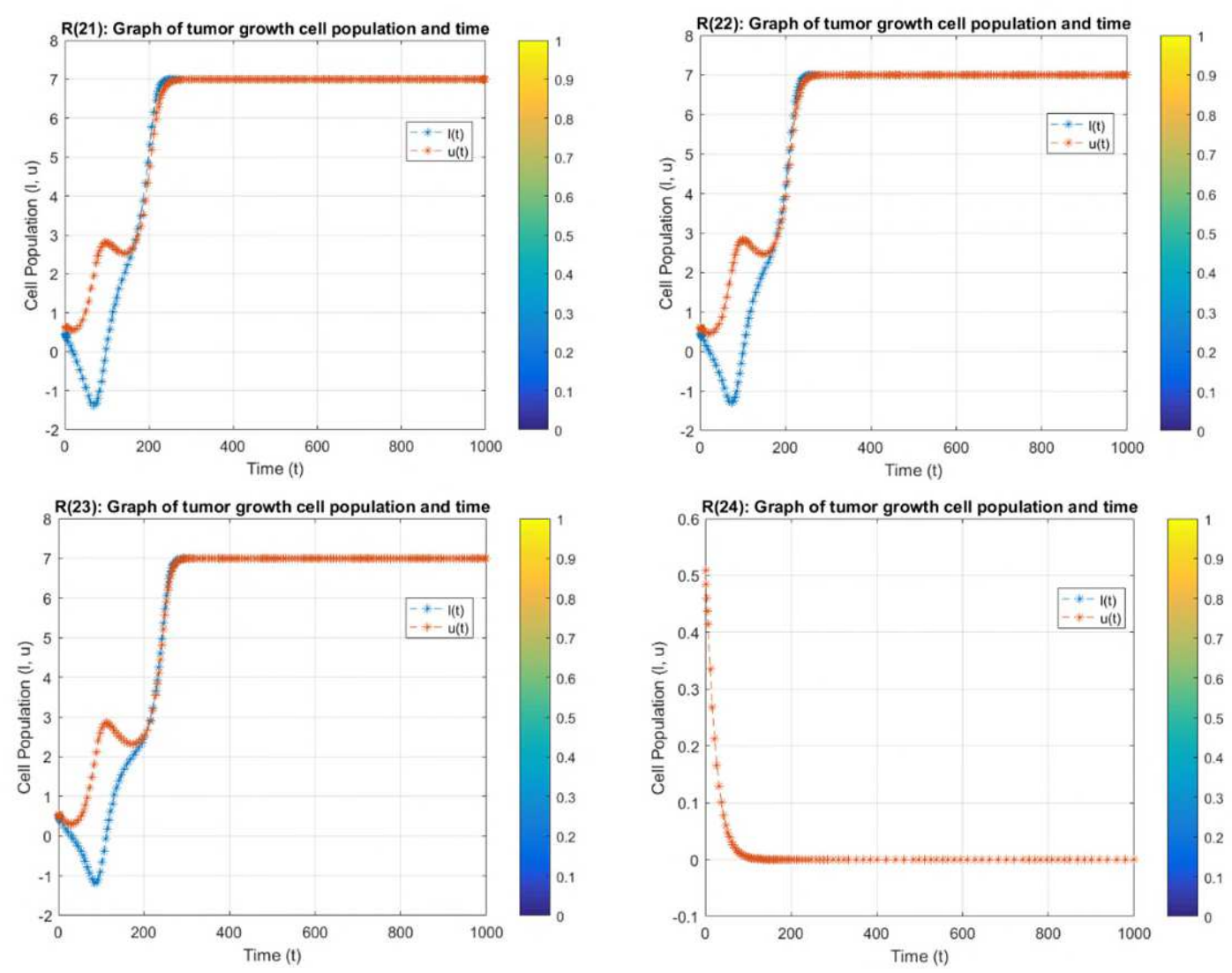

Fig. 3. Numerical simulation for both fuzzy initial condition and coefficient when $N(t)$ is (ii)$\mathrm{gH}$ for tumor growth model with Allee effect. For $\alpha=0, \alpha=0.3, \alpha=0.7$ the solution $N_{1}(t, \alpha) \geq N_{2}(t, \alpha)$ for $t \epsilon[0,200]$ and for $\alpha=1$, both $N_{1}(t, \alpha)=N_{2}(t, \alpha)$ for all $t$ and finally 
approaches to the unstable solution. This figure also depicted a Simultaneous graph of the tumor growth model when the Initial value is greater or smaller than the Allee threshold $(a=$ 2) and $K=7$.

\section{Comparative Analysis}
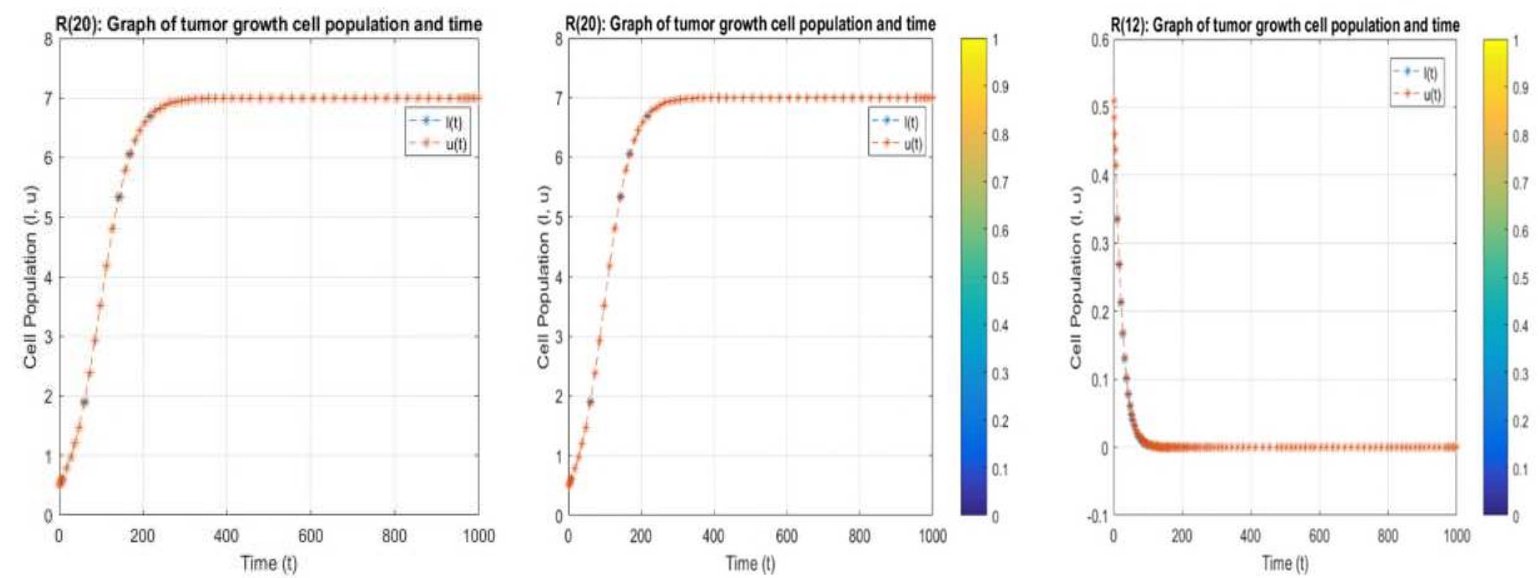

Fig. 4. demonstrates the comparative analysis of tumor modeling between crisp model and fuzzy model (comparison of the graph a and b) and model with or without Allee effect (comparison of graph $b$ and $c)$.

The crisp tumor growth model(graph a) explains the growth dynamics with the parameters as fixed which actually may not be but vary due to some reasons such as immune response, presence of resources such as nutrients and oxygen, etc. However, to analyze the model in a realistic way, fuzzy theory with the concept of membership function plays an important role in revealing the tumor growth dynamics properly. By taking the initial number of cell population and growth rate fuzzy in tumor shown in (graph b), one could be able to understand the behaviour tumor at a particular degree. Hence, target treatment easily. On the other side, graph ( $b$ and $c$ ) shows the mechanism with or without Allee effect under fuzzy environment. (graph b) demonstrates that without the Allee effect which is found to be relevant in tumor evolution, only tumor behaviour can be analyzed generally. However, the tumor model with the Allee effect (graph c) makes it possible to realize the situation at the onset of cancer as the initial condition and growth rate greatly affect the cancer dynamics with the Allee effect. The Allee effect's probable function brings up a slew of new avenues for comprehending and limiting tumor progression. 


\section{Results}

This current work displayed the tumour growth model with Allee effect under fuzzy environment using Generalized Hukuhara Derivative method. The result represents the two cases simultaneously through both i-gH (fig.2) and ii-gH (fig.3) derivative for different values of $\alpha$ $(\alpha=0,0.3,0.7$ and 1$)$. Case I: It is analyzed that for strong Allee effect $a=2$, the cell number will grow and tends to the carrying capacity $K$ and eventually attaining stability if the initial population is greater than the Allee threshold. Case II: while as, the cell number will decline towards 0 and may exhibit extinction. if the initial population is smaller than the Allee threshold. Hence, this all increase or decrease in cell number to a certain threshold makes it more appropriate to target the tumour treatment at early stages with suitable therapy.

The initial population and growth rate are greatly affected by the Allee effect as in the case of the higher cell population, there will be potentially high cooperative interaction and vice versa changes the growth rate accordingly. Fuzzy membership function clearly describes the mechanism of cancer growth with the Allee effect to a certain possibility degree. As in fig.2,3, at $\mu=0.4$, there is an increase in a cell population with an initial population greater than Allee threshold, and at $\mu=0.3$, there is decreasing trend of cell population with an initial population smaller than Allee threshold.

\section{Discussion and Conclusion}

For a better understanding of cancer dynamics, tumour growth modeling with the Allee effect in a fuzzy environment strongly confirms the occurrence of this ecological influence at the initiation of cancer. We explore the growth dynamics with the solution of the logistic equation using the derivative concept for various initial values and different growth rates. The involvement of Allee effect, the initial value and growth rate of the cell population have a significant impact on cancer dynamics. Depending on the purported grade function of the input, modeling imprecise initial conditions as well as coefficients around the critical with a crisp initial value might result in cell extinction to a certain degree.

The capacity to calculate and explain the nature of biological models with fuzzy parameter values, as well as solve all mathematical outcomes by numerical simulation with fuzzy parameters, demonstrates the resilience of the fuzzy from the ecological and mathematical 
perspective. Hence, it may be computed that the model's fuzziness makes the scenario more realistic because the parameter value cannot be known accurately. On the other side, in the presence of a strong Allee effect the administration of a maximum tolerated dose can be preferable to a therapy aiming just to control cancer in that the former can push the number of tumour cells below the threshold thus ensuring eradication. Hence, the occurrence of an Allee effect is also for the choice of the most appropriate therapy.

Fuzzy theory, Allee effect, and Generalized Hukuhara derivative concept successfully revealed the behaviour of tumor growth. With this developed model, new insights about tumor initiation and treatment are opened up at the true degree of freedom. Involvement of the Allee effect under the fuzzy environment in tumor treatment modeling will be the author's future research work. Furthermore, the different derivative concepts could be used to solve the tumor model under a fuzzy environment as fuzzy modeling reveals the realistic situation of tumor growth with the Allee effect.

Author contribution Rubeena Khaliq; Conceptualisation; Investigation; Writing (original draft prepration)

Pervaiz Iqbal; Conceptualization; Investigation; Supervision; Writing (review and editing)

Shahid Ahmad Bhat; Validation; Numerical Analysis; Supervision

Funding: Not applicable.

Data availability: Not applicable.

Declarations: All the author have declared the manuscript for publication.

Ethics approval and consent to participate: This article does not contain any studies with human participants or animals.

Consent for publication: All authors agree mutually with the participation and publication of the manuscript and declare that this is an original research.

Conflict of interest The authors declare no competing interest. 


\section{Refrences}

1. Hillen, T., \& Lewis, M. A. (2014). "Mathematical ecology of cancer. In Managing complexity, reducing perplexity". (pp. 1-13). Springer, Cham.

2. Pacheco, J. M., Santos, F. C., \& Dingli, D. (2014). "The ecology of cancer from an evolutionary game theory perspective". Interface focus, 4(4), 20140019.

3. Fouad, Y. A., \& Aanei, C. (2017). "Revisiting the hallmarks of cancer". American journal of cancer research, 7(5), 1016.

4. Korolev, K. S., Xavier, J. B., \& Gore, J. (2014). "Turning ecology and evolution against cancer". Nature Reviews Cancer, 14(5), 371-380.

5. Frank, S. A. (2007). Theory I. "In Dynamics of Cancer: Incidence, Inheritance, and Evolution”. Princeton University Press.

6. Nowak, M. A. (2006). "Evolutionary dynamics: exploring the equations of life". Harvard university press.

7. Nowell,P.C.(1976).“The clonal evolution of tumor cell populations”. Science, 194(4260), 2328.

8. Merlo, L. M., Pepper, J. W., Reid, B. J., \& Maley, C. C. (2006). "Cancer as an evolutionary and ecological process". Nature reviews cancer, 6(12), 924-935.

9. Crespi, B., \& Summers, K. (2005). "Evolutionary biology of cancer". Trends in ecology \& evolution, 20(10), 545-552.

10. Basanta, D., \& Anderson, A. R. (2013). "Exploiting ecological principles to better understand cancer progression and treatment". Interface focus, 3(4), 20130020.

11. Gatenby, R. A., Silva, A. S., Gillies, R. J., \& Frieden, B. R. (2009). "Adaptive therapy. Cancer" 1003 research 69, 4894-4903, doi: 10.1158/0008-5472 (Vol. 1004). CAN-08-3658.

12. Silva, A. S., Kam, Y., Khin, Z. P., Minton, S. E., Gillies, R. J., \& Gatenby, R. A. (2012). "Evolutionary approaches to prolong progression-free survival in breast cancer". Cancer research, 72(24), 6362-6370.

13. Basanta, D., Gatenby, R. A., \& Anderson, A. R. (2012). "Exploiting evolution to treat drug resistance: combination therapy and the double bind". Molecular pharmaceutics, 9(4), 914921.

14. Zahir, N., Sun, R., Gallahan, D., Gatenby, R. A., \& Curtis, C. (2020). "Characterizing the ecological and evolutionary dynamics of cancer". Nature Genetics, 52(8), 759-767. 
15. Whelan, C. J., \& Gatenby, R. A. (2020). "Special Collection on Ecological and Evolutionary Approaches to Cancer Control": Cancer Finds a Conceptual Home.

16. Ujvari, B., Papenfuss, A. T., \& Belov, K. (2016). "Transmissible cancers in an evolutionary context". Inside the cell, 1(1), 17-26.

17. Thomas, F., Fisher, D., Fort, P., Marie, J. P., Daoust, S., Roche, B., ... \& Hochberg, M. E. (2013). "Applying ecological and evolutionary theory to cancer: a long and winding road". Evolutionary Applications, 6(1), 1-10.

18. Taylor, T. B., Johnson, L. J., Jackson, R. W., Brockhurst, M. A., \& Dash, P. R. (2013). "First steps in experimental cancer evolution". Evolutionary applications, 6(3), 535-548.

19. Iwasa, Y., Michor, F., \& Nowak, M. A. (2004). "Evolutionary dynamics of invasion and escape". Journal of Theoretical Biology, 226(2), 205-214.

20. Somarelli, J. A., Gardner, H., Cannataro, V. L., Gunady, E. F., Boddy, A. M., Johnson, N. A., \& Townsend, J. P. (2020). "Molecular biology and evolution of cancer: from discovery to action". Molecular biology and evolution, 37(2), 320-326.

21. Bergman, A., \& Gligorijevic, B. (2015). "Niche construction game cancer cells play". The European Physical Journal Plus, 130(10), 1-7.

22. Maley, C. C., Aktipis, A., Graham, T. A., Sottoriva, A., Boddy, A. M., Janiszewska, M., \& Shibata, D. (2017). "Classifying the evolutionary and ecological features of neoplasms". Nature Reviews Cancer, 17(10), 605.

23. Adler, F. R., \& Gordon, D. M. (2019). "Cancer ecology and evolution: positive interactions and system vulnerability". Current opinion in systems biology, 17, 1-7.

24. Pashov, A., Hernandez Puente, C. V., Ibrahim, S. M., Monzavi-Karbassi, B., Makhoul, I., \& Kieber-Emmons, T. (2018). "Thinking cancer". Monoclonal antibodies in immunodiagnosis and immunotherapy, 37(3), 117-125.

25. Enderling, H., \& AJ Chaplain, M. (2014). "Mathematical modeling of tumor growth and treatment". Current pharmaceutical design, 20(30), 4934-4940.

26. Courchamp, F., Clutton-Brock, T., \& Grenfell, B. (1999). "Inverse density dependence and the Allee effect". Trends in ecology \& evolution, 14(10), 405-410.

27. Kramer, A. M., Dennis, B., Liebhold, A. M., \& Drake, J. M. (2009). "The evidence for Allee effects". Population Ecology, 51(3), 341-354. 
28. Courchamp, Franck, Tim Clutton-Brock, and Bryan Grenfell. (1999). "Inverse density dependence and the Allee effect." Trends in ecology \& evolution 14.10: 405-410.

29. Sun, G. Q. (2016). "Mathematical modeling of population dynamics with Allee effect". Nonlinear Dynamics, 85(1), 1-12.

30. Merdan, H., et al. (2009). "Allee effects on population dynamics in continuous (overlapping) case." Chaos, Solitons \& Fractals 39.4: 1994-2001.

31. Çelik, C., et al. (2008)."Allee effects on population dynamics with delay." Chaos, Solitons \& Fractals 37.1: 65-74.

32. Fowler, M. S., and G. D. Ruxton. (2002). "Population dynamic consequences of Allee effects." Journal of Theoretical Biology 215.1: 39-46.

33. Hadjiavgousti, Despina, and Simos Ichtiaroglou. (2004)."Existence of stable localized structures in population dynamics through the Allee effect." Chaos, Solitons \& Fractals 21.1: 119-131.

34. López-Ruiz, Ricardo, and Danièle Fournier-Prunaret. (2005). "Indirect Allee effect, bistability and chaotic oscillations in a predator-prey discrete model of logistic type." Chaos, Solitons \& Fractals 24.1: 85-101.

35. Delitala, M., \& Ferraro, M. (2020). "Is the Allee effect relevant in cancer evolution and therapy"? AIMS Mathematics, 5(6), 7649-7660.

36. Benzekry, S., Lamont, C., Beheshti, A., Tracz, A., Ebos, J. M., Hlatky, L., \& Hahnfeldt, P. (2014). "Classical mathematical models for description and prediction of experimental tumor growth". PLoS Comput Biol, 10(8), e1003800.

37. Johnson, K. E., Howard, G., Mo, W., Strasser, M. K., Lima, E. A., Huang, S., \& Brock, A. (2019). "Cancer cell population growth kinetics at low densities deviate from the exponential growth model and suggest an Allee effect". PLoS biology, 17(8), e3000399.

38. Neufeld, Z., von Witt, W., Lakatos, D., Wang, J., Hegedus, B., \& Czirok, A. (2017). "The role of Allee effect in modelling post resection recurrence of glioblastoma". PLoS computational biology, 13(11), e1005818.

39. Courchamp, F., Berec, L., \& Gascoigne, J. (2008). "Allee effects in ecology and conservation". Oxford University Press. 
40. Böttger, K., Hatzikirou, H., Voss-Böhme, A., Cavalcanti-Adam, E. A., Herrero, M. A., \& Deutsch, A. (2015). "An emerging Allee effect is critical for tumor initiation and persistence". PLoS Comput Biol, 11(9), e1004366.

41. Feng, P., Dai, Z., \& Wallace, D. (2019). “On a 2d model of avascular tumor with weak allee effect”. Journal of Applied Mathematics, 2019.

42. Jafelice, R. M., Silva, C. A., Barros, L. C., \& Bassanezi, R. C. (2015). "A fuzzy delay approach for HIV dynamics using a cellular automaton". Journal of Applied Mathematics, 2015.

43. Bhat, S. A. (2020). "Some Methods for Prioritization of Alternatives of MADM Problems In Fuzzy Environment And Its Extensions” (Doctoral dissertation).

44. Singh, A., \& Bhat, S. (2020). A note on "A novel correlation coefficient of intuitionistic fuzzy sets based on the connection number of set pair analysis and its application". Authorea Preprints.

45. Ahmadi, Hossein, et al. (2018)."Diseases diagnosis using fuzzy logic methods: A systematic and meta-analysis review." Computer Methods and Programs in Biomedicine 161: 145-172.

46. Esmaili, S. S., \& Nasrabadi, A. M. (2009, December). "Uncertainty analysis in tumor model with fuzzy parameters”. In 2009 International Conference on Computational Intelligence and Software Engineering (pp. 1-4). IEEE.

47. Esmaili, S. S., \& Nasrabadi, A. M. (2010). "Different initial conditions in fuzzy Tumor model”. Journal of Biomedical Science and Engineering, 3(10), 1001.

48. Rivaz, A., Azizian, M., Kamyad, A. V., \& Zadeh, S. Z. (2018). “A full fuzzy generalized mathematical model of tumor growth and its analysis". Journal of Intelligent \& Fuzzy Systems, 35(6), 6453-6460.

49. Souza, T. R., Mancera, P. F., \& Bassanezi, R. C. (2020). "Dynamics of tumor growth: chemotherapy and integrative oncology". Computational and Applied Mathematics, 39(1), 118.

50. d'Onofrio, A. (2008). "Fuzzy oncology": Fuzzy noise induced bifurcations and their application to anti-tumor chemotherapy. Applied mathematics letters, 21(7), 662-668.

51. Gholami, S., Alasty, A., Salarieh, H., \& Hosseinian-Sarajehlou, M. (2015). "On the control of tumor growth via type-1 and interval type-2 fuzzy logic". Journal of Mechanics in Medicine and Biology, 15(05), 1550083. 
52. Nasiri, H., \& Kalat, A. A. (2018). "Adaptive fuzzy back-stepping control of drug dosage regimen in cancer treatment”. Biomedical Signal Processing and Control, 42, 267-276.

53. Obajemu, O., Mahfouf, M., \& Catto, J. W. (2017). “A new fuzzy modeling framework for integrated risk prognosis and therapy of bladder cancer patients". IEEE Transactions on Fuzzy Systems, 26(3), 1565-1577.

54. Razmi, F., Moghaddam, R. K., \& Rowhanimanesh, A. (2015). "Control of cancer growth using two input autonomous fuzzy nanoparticles”. Nano, 10(04), 1550062.

55. Uzun, B., \& Kiral, E. (2017). "Application of markov chains-fuzzy states to gold price". Procedia computer science, 120, 365-371.

56. Stefanini, Luciano, and Barnabas Bede. (2009). "Generalized Hukuhara differentiability of interval-valued functions and interval differential equations." Nonlinear Analysis: Theory, Methods \& Applications 71.3-4: 1311-1328.

57. Bede, B. (2008). Note on "Numerical solutions of fuzzy differential equations by predictorcorrector method". Information sciences, 178(7), 1917-1922.

58. Ezadi, S., \& Allahviranloo, T. (2017). "Numerical solution of linear regression based on Znumbers by improved neural network". Intelligent Automation \& Soft Computing, 1-11.

59. Salahshour, S., Ahmadian, A., Mahata, A., Mondal, S. P., \& Alam, S. (2018). "The behavior of logistic equation with alley effect in fuzzy environment: fuzzy differential equation approach". International Journal of Applied and Computational Mathematics, 4(2), 1-20. 